\title{
OPTIMAL LINEAR EXTENSIONS BY INTERCHANGING CHAINS
}

\author{
IVAN RIVAL
}

\begin{abstract}
For a finite ordered set $P$ how can a linear extension $L=C_{1} \oplus C_{2}$ $\oplus \cdots \oplus C_{m}$ be constructed which minimizes the number $m$ of chains $C_{i}$ of $P$ ? While this question remains largely unanswered we show that a natural "greedy" algorithm is actually optimal for a far wider class of ordered sets than was hitherto suspected.
\end{abstract}

The most fundamental and yet far-reaching results in the theory of ordered sets are the following:

(R. P. Dilworth [1950]) In a finite ordered set $P$, the minimum number of disjoint chains whose set union is all of $P$ equals the maximum number of pairwise noncomparable elements of $P$.

(E. Szpilrajn [1930]) Every ordered set has a linear extension.

Let $C_{1}, C_{2}, \ldots, C_{m}$ be any minimum family of disjoint chains of a finite ordered set $P$ whose set union is $P$. The linear sum $C_{1} \oplus C_{2} \oplus \cdots \oplus C_{m}$ of these chains need not be a linear extension of $P$. On the other hand, any linear extension $L$ of a finite ordered set $P$ can be expressed as the linear sum $C_{1} \oplus C_{2} \oplus \cdots \oplus C_{m}$ of chains $C_{i}$ in $P$ (so chosen that $\sup _{P} C_{i} \$ \inf _{P} C_{i+1}$ for each $i$ ) and, while $\cup_{i=1}^{k} C_{i}=P$, this family of chains need not be the minimum number whose set union is $P$ (see Figure 1). How can these two fundamental results about ordered sets be reconciled? Indeed, until recently there seemed little reason even to consider such a question.

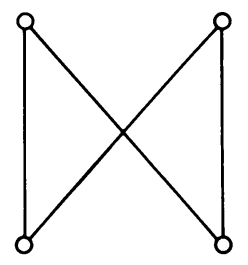

FIGURE 1

Suppose we are to schedule a set of jobs for processing, one at a time, by a single machine. Precedence constraints, due perhaps to technological dictates, prohibit the start of certain jobs until certain other jobs are already completed. A job which is performed immediately after a job which is not constrained to precede it requires a

Received by the editors September 16, 1982 and, in revised form, February 16, 1983.

1980 Mathematics Subject Classification. Primary 06A10, 06A05; Secondary 90B35.

Key words and phrases. Linear extension, chain decomposition. 
"setup"-entailing some additional cost. We consider the simplest variation: "schedule the jobs to minimize the number of setups". In the language of ordered sets this is commonly rendered as follows. Let $P$ be a finite ordered set and let $\mathcal{L}(P)$ stand for all of its linear extensions. For $L \in \mathcal{L}(P)$ let $s(P, L)$ count the number of pairs $(a, b)$ of elements $a, b$ of $P$ such that $a$ covers $b$ in $L$ (that is, $a>x \geqslant b$ implies $x=b)$, and $a \neq b$ in $P$. Each such pair $(a, b)$ is called a setup of $L$. Put

$$
s(P)=\min \{s(P, L) \mid L \in \mathcal{L}(P)\}
$$

the setup number of $P$ (see Figure 2). The problem is this: Construct $L \in \mathcal{L}(P)$ for which $s(P, L)=s(P)$. It is considered in several recent articles (cf. G. Chaty and M. Chein [1979], M. Chein and M. Habib [1980], O. Cogis and M. Habib [1979], D. Duffus, I. Rival, and P. M. Winkler [1982], G. Gierz and W. Poguntke [1982], M. Habib [1981], W. R. Pulleyblank [1982]). The purpose of this note is to prove that a natural "greedy" linear extension is actually optimal for a far wider class of ordered sets than was hitherto suspected.
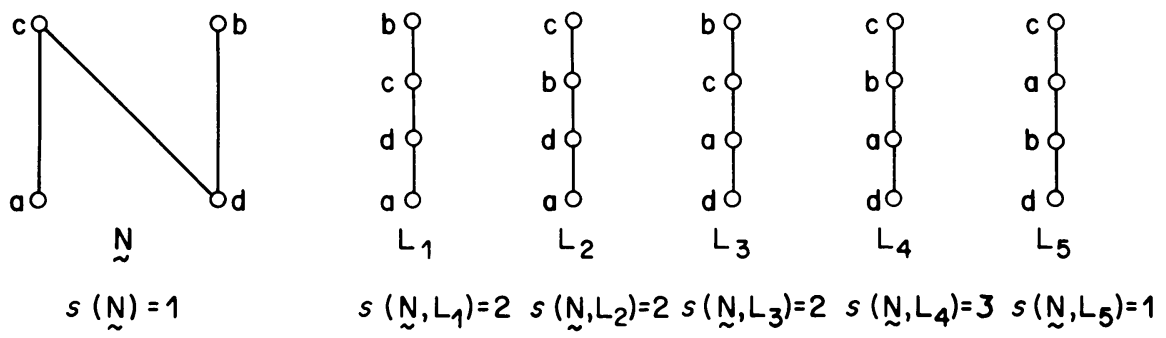

FigURE 2

TheOREM. Let $P$ be a finite ordered set. Then, for any greedy linear extension $L$ of $P$, $s(P)=s(P, L)$ whenever $P$ is $\mathbf{N}$-free.

$\mathbf{N}$ stands for the ordered set illustrated in Figure 2 ; that is, $\mathbf{N}=\{a, b, c, d\}$ subject to $a<c, d<c, d<b, a \nless b, b \nless c, d \nless a$ (see also Figure 3(d)). The "diagrams" of the ordered sets illustrated in Figure 3 should clarify just when an ordered set is "N-free": each of the ordered sets (a), (c), (e), (g) is N-free while none of (b), (d), (f) is. An ordered set $P$ is called $\mathbf{N}$-free if it contains no cover-preserving subset isomorphic to $\mathbf{N}$. Examples of $\mathbf{N}$-free ordered sets abound. For instance, any series-parallel ordered set is $\mathbf{N}$-free. A reason for this is that a series-parallel ordered set contains no subset isomorphic to $\mathbf{N}$ at all (cf. J. Valdes [1978], J. Valdes, R. E. Tarjan and E. L. Lawler [1982], W. R. Pulleyblank [1982]). So, for example, the ordered set in Figure 3(e) is $\mathbf{N}$-free although it is not series-parallel. The series-parallel character of an ordered set $P$ is determined by examining the directed comparability graph of $\boldsymbol{P}$ for $\mathbf{N}$ 's while the $\mathbf{N}$-free character of $P$ is determined by examining its directed covering graph (that is, its diagram) for $\mathbf{N}$ 's. But $\mathbf{N}$-free ordered sets constitute a much wider class of ordered sets than series-parallel ordered sets. For instance, while every subset of a series-parallel ordered set must itself be series-parallel, every finite ordered set can be embedded in an $\mathbf{N}$-free ordered set! 


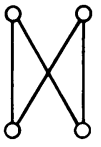

(a)

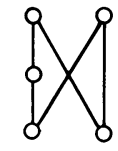

(b)

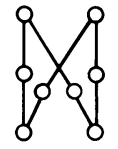

(c)

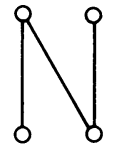

(d)

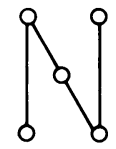

(e)

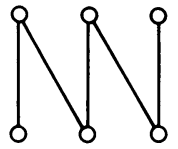

(f)

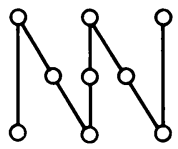

(g)

FIGURE 3

The other ingredient of our theorem is a "greedy" linear extension. Loosely speaking, this is any linear extension constructed as follows. Choose $a_{1} \in P$ maximal such that $C_{1}=\left\{x \in P \mid x \leqslant a_{1}\right\}$ is a chain in $P$; choose $a_{2} \in P-C_{1}$ maximal such that $C_{2}=\left\{x \in P-C_{1} \mid x \leqslant a_{2}\right\}$ is a chain in $P-C_{1}$; in general, choose $a_{i} \in P-$ $\cup_{j<i} C_{j}$ maximal such that $C_{i}=\left\{x \in P-\cup_{j<i} C_{j} \mid x \leqslant a_{i}\right\}$ is a chain in $P-$ $\cup_{j<i} C_{j}$. Eventually $P=\cup_{j=1}^{m} C_{j}$ for some $m$ and $L=C_{1} \oplus C_{2} \oplus \cdots \oplus C_{m}$ is a linear extension of $P$. For example, for the ordered set $\mathbf{N}$, as illustrated in Figure 2, each of the linear extensions $L_{1}, L_{2}, L_{5}$ is greedy, while neither $L_{3}$ nor $L_{4}$ is. A linear extension $L$ of $P$ is a greedy linear extension if, for some $m, L=C_{1} \oplus C_{2} \oplus \cdots \oplus C_{m}$, where each $C_{i}$ is a chain in $P$, each $\sup _{P} C_{i} \$ \inf _{P} C_{i+1}$, and for each $i$ and for any $x \in P$ with $\sup _{P} C_{i}<x$ in $P$, there is a $y \in \cup_{j=i+1}^{m} C_{j}$ such that $y<x$ in $P$. (For each $i$ and any linear extension $L^{\prime}$ of $P$ with initial segment $I=C_{1} \oplus C_{2} \oplus \cdots \oplus C_{i}$, $\left(\sup _{L^{\prime}} I, \inf _{L^{\prime}}(P-I)\right)$ is a setup of $L^{\prime}$.) O. Cogis and M. Habib [1979] have observed that, for any series-parallel ordered set $P$ and any greedy linear extension $L$ of $P$, $s(P)=s(P, L)$. Actually, for any finite ordered set $P$ there is a greedy linear extension $L$ of $P$ satisfying $s(P)=s(P, L)$. For some ordered sets greedy linear extensions are far from "optimal": the setup number for the direct product of the two-element chain with an $n=$ element chain is 1 , yet there is a greedy linear extension with $n-1$ setups. For some ordered sets there are "optimal" linear extensions which are not greedy (see Figure 4). On the other hand there are ordered sets $P$ for which $s(P)=s(P, L)$ for every greedy linear extension, yet $P$ is not $\mathbf{N}$-free (see Figure 5) (cf. O. Cogis [1982]).

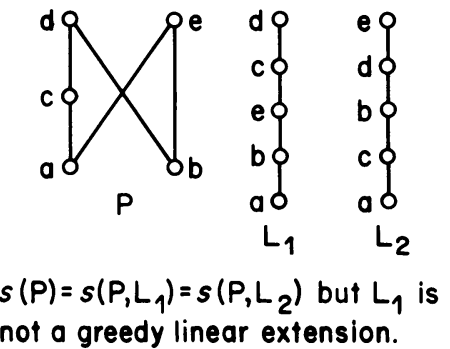

FIGURE 4

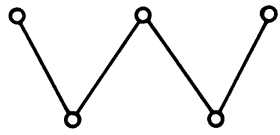

FIGURE 5

A final remark will be made before we get to the proof of the theorem. There are several scheduling problems for which efficient algorithms are known provided the precedence constraints satisfy certain special conditions. The most common such condition is series-parallel. Insofar as the class of $\mathbf{N}$-free ordered sets constitutes a far broader class than the class of series-parallel ordered sets, and insofar as the 
greedy linear extension is efficient and optimal for each $\mathbf{N}$-free ordered set-at least for the setup number problem - it may be that efficient algorithms for some other scheduling problems will work even for $\mathbf{N}$-free ordered sets. The efficiency of algorithms for series-parallel ordered sets is usually linked to the simple decomposition rules associated with "series" and "parallel" constructions. While there seems to be no obvious analogue of these constructions for $\mathbf{N}$-free ordered sets it may be worth recalling this result of P. Grillet [1969]: a finite ordered set is $\mathbf{N}$-free if and only if each maximal chain intersects each maximal antichain (cf. B. Leclerc and B. Monjardet [1973]).

The proof. Here is our approach. Let $L_{0}=\left\{x_{1}<x_{2}<\cdots<x_{n}\right\}$ be any optimal linear extension of $P$; that is, $s\left(P, L_{0}\right)=s(P)$. Let $L_{g}=\left\{y_{1}<y_{2}<\cdots<y_{n}\right\}$ be any greedy linear extension of $P$. Let $i$ be the least index such that $y_{i} \neq x_{i}$; put $a=x_{i}$. Then for some $k>i, y_{k}=a$. Let $b=y_{k-1}$. If $a>b$ in $P$ then, for some $r<i, x_{r}=b=y_{k-1}$ and, as $k-1 \geqslant i>r, x_{r} \neq y_{r}$ which contradicts the assumption that $x_{r}=y_{r}$ for each $r=1,2, \ldots, i-1$. Therefore $a \neq b$ in $P$ and $(a, b)$ is a setup of $L_{g}$. Now, let $A=\left\{a=y_{k}<y_{k+1}<\cdots<y_{l}\right\}$ be a chain of $P$ and of $L_{g}$ and maximal such that $b \nless y_{l}$ in $P$. Dually, let $B=\left\{b=y_{k-1}>y_{k-2}>\cdots>y_{j}\right\}$ be a chain of $P$ and of $L_{g}$ and maximal such that $a \ngtr y_{j}$ in $P$. Set

$$
L_{g}(a / b)=\left\{y_{1}<y_{2}<\cdots<y_{j-1}\right\} \oplus A \oplus B \oplus\left\{y_{l+1}<y_{l+2}<\cdots<y_{n}\right\},
$$

a "chain interchange" of $L_{g}$ (cf. Figure 6). Then $L_{g}(a / b)$ satisfies these conditions:

(i) $L_{g}(a / b)$ is a greedy linear extension of $P$;

(ii) $s\left(P, L_{g}(a / b)\right)=s\left(P, L_{g}\right)$.

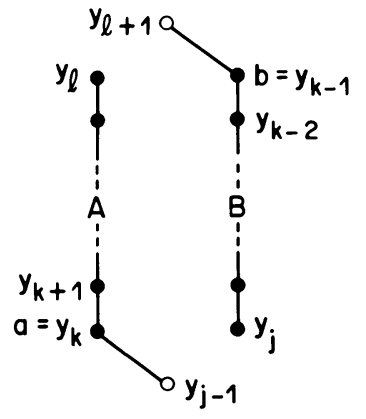

P

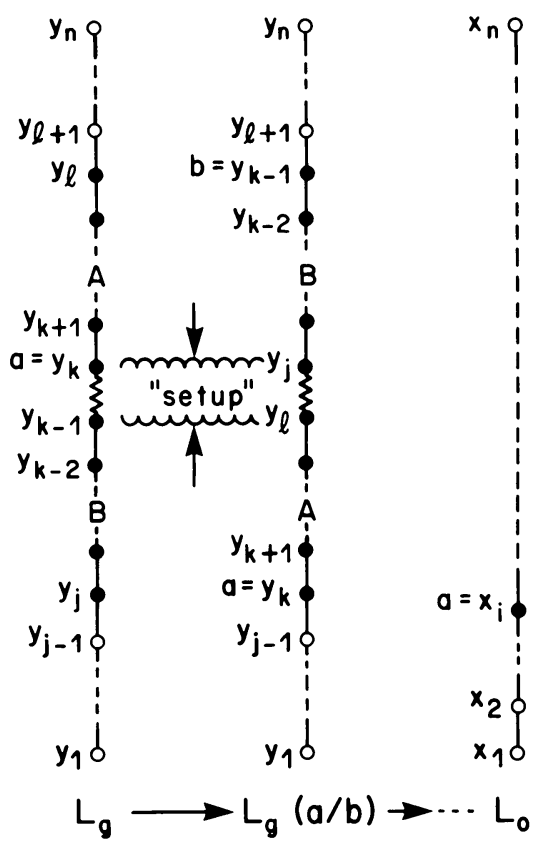

Figure 6 
Also, once we relabel $L_{g}(a / b)=\left\{w_{1}<w_{2}<\cdots<w_{n}\right\}$ then $a=w_{k^{\prime}}$ for $i \leqslant k^{\prime}<k$. This means that a sequence of "chain interchanges" will transform $L_{g}$ into $L_{g}^{\prime}=\left\{z_{1}\right.$ $\left.<z_{2}<\cdots<z_{n}\right\}$ for which the least index $s$ such that $z_{s} \neq x_{s}$ is strictly greater than $i$. It follows that a sequence of "chain interchanges" will transform $L_{g}$ into $L_{0}$ and, in view of (ii), $s\left(P, L_{g}\right)=s\left(P, L_{0}\right)=s(P)$, which is our claim.

It will be convenient to use some additional abbreviations. Let $a, b \in P$ and let $L \in \mathcal{L}(P)$. If $a>b$ in $P$ we can also write $a>b(P)$ and so if $a \ngtr b$ in $P$ but $a>b$ in $L$ we write $a \ngtr b(P)$ and $a>b(L)$. For $a$ covers $b$ we write $a>b$, as is the custom. Let $\mathbf{N}(P)$ stand for the set of all cover-preserving $\mathbf{N}$ 's in $P$ with the additional convention that members of $\mathbf{N}(P)$ are written as ordered four-tuples $(a, c, d, b)$, where $a \prec c(P), c \succ d(P), d \prec b(P), a \nless b(P), b \nless c(P), d \nless a(P)$ (cf. Figure 2).

The idea which inspired our theorem is contained in this lemma.

N-Lemma. Let $P$ be a finite ordered set, let $a, b \in P$, and let $L \in \mathcal{L}(P)$ such that $a>b(L)$ but $a \neq b(P)$. Then either there is an $L^{\prime} \in \mathcal{L}(P)$ satisfying $a<b\left(L^{\prime}\right)$ and $s\left(P, L^{\prime}\right) \leqslant s(P, L)$ or else $\left(a^{\prime}, c, d, b^{\prime}\right) \in \mathbf{N}(P)$ for some $a \leqslant a^{\prime} \prec c$ and $b \geqslant b^{\prime} \succ d$.

Proof. Let $A$ be a chain in $P \cap L$ with least element $a$ and maximal so that $x \in A$ implies $x \neq b(P)$. Let $B$ be a chain in $P \cap L$ with greatest element $b$ and maximal so that $y \in B$ implies $y \neq a(P)$. If we write $L$ as $D \oplus B \oplus A \oplus C$ then we construct $L^{\prime}=D \oplus A \oplus B \oplus C$.

Is $L^{\prime} \in \mathcal{L}(P)$ ? If not, there must be $x \in A$ and $y \in B$ such that $x>y(P)$. In this case let

$$
c=\inf _{P}\{x \in A \mid x>y(P) \text { some } y \in B\} \quad \text { and } \quad d=\sup _{P}\{y \in B \mid y<c(P)\} .
$$

According to the construction of $A$ and $B, c>a(P)$ and $d\langle b(P)$. If $c>z \geqslant d(P)$ then, according to the construction of $c, z \notin A$. But then $z \in B$ and, according to the construction of $d, z=d$. This shows that $c>d(P)$. Let $a^{\prime} \in A$ satisfy $a^{\prime} \prec c(L)$ and let $b^{\prime} \in B$ satisfy $d \prec b^{\prime}(L)$. Then $\left(a^{\prime}, c, d, b^{\prime}\right) \in \mathbf{N}(P)$.

Let us suppose then that $L^{\prime} \in \mathcal{L}(P)$. Is $s\left(P, L^{\prime}\right) \leqslant s(P, L)$ ? To analyse this let $a^{\prime} \succ \sup _{L} A(L)$ and let $b^{\prime} \prec \inf _{L} B(L)$. Then, apart from the $\operatorname{setup}\left(\inf _{L} B, \sup _{L} A\right)$ in $L^{\prime}$ (which corresponds to the setup $(a, b)$ in $L$ ) the only possible setups in $L^{\prime}$, which can be different from those in $L$, are $\left(a^{\prime}, b\right)$ and $\left(a, b^{\prime}\right)$. Suppose $a^{\prime} \ngtr b(P)$ but $a^{\prime}>\sup _{L} A(P)$. From the construction of $A$, it must be that $a^{\prime}>b(P)$. It follows that if $\left(a^{\prime}, b\right)$ is a setup in $L^{\prime}$ then $\left(a^{\prime}, \sup _{L} A\right)$ is a setup in $L$. Similarly, if $\left(a, b^{\prime}\right)$ is a setup in $L^{\prime}$ then $\left(\inf _{L} B, b^{\prime}\right)$ is a setup in $L$. Therefore, $s\left(P, L^{\prime}\right) \leqslant s(P, L)$.

Suppose $L$ is a linear extension of an ordered set $P$ and $(a, b)$ is a setup of $L$. If there is no $\left(a^{\prime}, c, d, b^{\prime}\right) \in \mathbf{N}(P)$, where $a \leqslant a^{\prime} \prec c(P)$ and $b \geqslant b^{\prime}>d(P)$, then the linear extension $L^{\prime}$ constructed in the $\mathbf{N}$-lemma is said to be obtained from $L$ by interchanging chains; we denote it by $L(a / b)$. (Such a linear extension $L(a / b)$, if it exists, is uniquely determined by the setup $(a, b)$.) According to the N-lemma $s(P, L(a / b)) \leqslant s(P, L)$ and, actually, this inequality can be strict. For example, the 
linear extension $L_{3}$ of $\mathbf{N}$ illustrated in Figure 2 has the setup $(b, c) . L_{5}$ is obtained from $L_{3}$ by interchanging chains $\left(L_{5}=L_{3}(b / c)\right)$ and $s\left(\mathbf{N}, L_{5}\right)<s\left(\mathbf{N}, L_{3}\right)$. However, $L_{3}$ is not a greedy linear extension of $\mathbf{N}$ !

LEMMA 2. Let $P$ be a finite ordered set and let $L$ be a greedy linear extension of $P$. Let $a>b(L)$ but $a \ngtr b(P)$ and let $L(a / b) \in \mathcal{L}(P)$ be obtained from $L$ by interchanging chains. Then either $s(P, L(a / b))=s(P, L)$ or else $\left(b, a^{\prime}, c, c^{\prime}\right) \in \mathbf{N}(P)$ where $a^{\prime}>\sup _{L} A(L)$ and $a \leqslant c \prec c^{\prime} \leqslant \sup _{L} A(P)$.

Proof. Let us write $L=C_{1} \oplus C_{2} \oplus \cdots \oplus C_{m}$ where each $C_{i}$ is a chain in $P$ and each $\sup _{P} C_{i} \neq \inf _{P} C_{i+1}(P)$. Suppose $s(P, L(a / b))<s(P, L)$. From the construction in the $\mathbf{N}$-lemma this means that either $\left(a^{\prime}, \sup _{L} A\right)$ is a setup in $L$, where $a^{\prime}>\sup _{L} A(L)$ and $a^{\prime}>b(P)$, or else $\left(\inf _{L} B, b^{\prime}\right)$ is a setup in $L$, where $\inf _{L} B>$ $b^{\prime}(L)$ and $a>b^{\prime}(P)$.

Suppose first that $\left(a^{\prime}, \sup _{L} A\right)$ is a setup in $L$ and $a^{\prime}>b(P)$. As $L$ is a greedy linear extension and $a^{\prime}>b=\sup _{L} C_{i}(P)$ for some $i$, there must be $c \prec a^{\prime}(P)$ such that $c \notin \cup_{j<i} C_{j}$. Then $c \in A$. Also, $c<\sup _{L} A(L)\left(\right.$ since $c=\sup _{L} A$ implies $c \nless$ $\left.a^{\prime}(P)\right)$. Then it is easy to verify that $\left(b, a^{\prime}, c, c^{\prime}\right) \in \mathbf{N}(P)$ where $c \prec c^{\prime} \leqslant \sup _{L} A(P)$.

Suppose now that $\left(\inf _{L} B, b^{\prime}\right)$ is a setup in $L$ and $a>b^{\prime}(P)$. (Note that this is not excluded on grounds of duality because greedy linear extension is not a self-dual concept.) As $L$ is a greedy linear extension and $b^{\prime}=\sup _{L} C_{k} \nless \inf _{L} B(L)$, for some $k$, there must be $y \prec a(P)$ such that $y \notin \cup_{j \leqslant k} C_{j}$. Then $y \in B$, which, however, is impossible in view of the construction of $B$ for $L(a / b)$.

LEMMA 3. Let $P$ be a finite, $\mathbf{N}$-free ordered set. If $L$ is a greedy linear extension of $P$ and $(a, b)$ is a setup in $L$ then the linear extension $L(a / b)$ obtained from $L$ by interchanging chains is greedy.

Proof. Again we express the linear extensions $L$ and $L(a / b)$ as linear sums of chains in $P$, that is,

$$
L=C_{1} \oplus C_{2} \oplus \cdots \oplus C_{m}
$$

where each $C_{i}$ is a chain in $P$ and each $\sup _{P} C_{i} \neq \inf _{P} C_{i+1}(P)$ and

$$
L(a / b)=C_{1}^{\prime} \oplus C_{2}^{\prime} \oplus \cdots \oplus C_{m}^{\prime}
$$

where each $C_{i}^{\prime}$ is a chain in $P$ and each $\sup _{P} C_{i}^{\prime} \neq \inf _{P} C_{i+1}^{\prime}(P)$. (Note that $s(P, L(a / b))=s(P, L)$ is a consequence of Lemma 2.) Suppose now that $L(a / b)$ is not a greedy linear extension. Then, for some $i$, and for some $v \in P-\cup_{j \leqslant i} C_{j}^{\prime}$, $v>\sup _{P} C_{i}^{\prime}(P)$ and each $x \prec v(P)$ satisfies $x \in \cup_{j<i} C_{j}^{\prime}$.

Suppose $\sup _{P} C_{i}^{\prime}<b^{\prime}(L(a / b))$ where $b^{\prime} \prec \inf _{L} B(L)$. Then, according to the construction of $L(a / b), C_{j}^{\prime}=C_{j}$ for each $j=1,2, \ldots, i$. This is impossible, though, since $L$ is a greedy linear extension. Therefore $\sup _{P} C_{i}^{\prime} \geqslant b^{\prime}(L(a / b))$.

Suppose $\sup _{P} C_{i}^{\prime}=b^{\prime}$. Then $a \ngtr b^{\prime}(P)$ although, of course, $a \succ b^{\prime}(L(a / b))$. As $s(P, L(a / b))=s(P, L), \inf _{L} B \ngtr b^{\prime}(P)$ although $\inf _{L} B \succ b^{\prime}(L)$. This implies that $C_{i}^{\prime}=C_{i}$ again, and this too contradicts the choice of $L$ as a greedy linear extension. Then $\sup _{P} C_{i}^{\prime}>b^{\prime}(L(a / b))$. 
Suppose $\sup _{P} C_{i}^{\prime}=\sup _{L} A$. Then as $L$ is a greedy linear extension $a^{\prime}>\sup _{L} A(P)$ where $a^{\prime} \succ \sup _{L} A(L)$. As $s(P, L(a / b))=s(P, L)$ this means that $a^{\prime} \succ b(P)$. Now, $v \neq a^{\prime}$ and $v \neq b(P)$ (Right?). Then $\left(b, a^{\prime}, \sup _{L} A, v\right) \in \mathbf{N}(P)$ which is impossible.

Suppose then that $\sup _{P} C_{i}^{\prime}>\sup _{L} A(L(a / b))$. Again, $\sup _{P} C_{i}^{\prime}>b(L(a / b))$ violates the assumption that $L$ is a greedy linear extension. So there remains the case that $\sup _{P} C_{i}^{\prime}=b$. Then $a^{\prime}>b(L(a / b))$ but $a^{\prime} \ngtr b(P)$ where $a^{\prime}>\sup _{L} A(L)$. As $s(P, L(a / b))=s(P, L), a^{\prime} \ngtr \sup _{L} A(P)$. Now $v \geqslant a^{\prime}(L)$ and as $L$ is a greedy linear extension there is $x \prec v(P)$ such that $x \nless b(L)$ although $x<b(L(a / b))$. Therefore, $x \in A$. If $x<\sup _{L} A(P)$ then $(b, v, x, c) \in \mathbf{N}(P)$ where $x<c \leqslant$ $\sup _{L} A(P)$. Otherwise, $x=\sup _{L} A$. As $L$ is a greedy linear extension there must be $x^{\prime} \prec v(P)$ such that $x^{\prime}>\sup _{L} A(L)$ although, by assumption $x^{\prime} \in \cup_{j \leqslant i} C_{j}^{\prime}$.

The proof of the theorem is now an immediate consequence of the $\mathbf{N}$-lemma and Lemma 3.

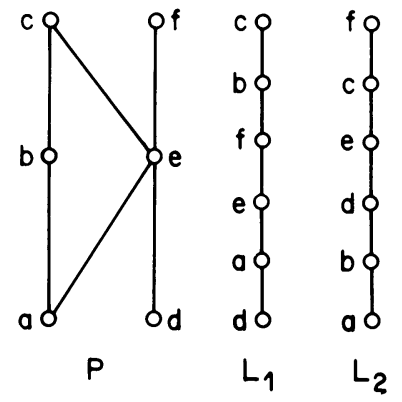

FigURE 7

Two final remarks are in order. The first is that for a finite, $\mathbf{N}$-free ordered set every optimal linear extension must be greedy. This follows from Lemma 3. As we have observed earlier (cf. Figure 4) this is not true of an arbitrary finite ordered set.

The next remark is concerned with the potential use of this procedure of interchanging chains. For a finite, $\mathbf{N}$-free ordered set any "optimal" linear extension can be obtained from any other "optimal" linear extension by a sequence of linear extensions each constructed by interchanging chains. This is no longer true for an arbtrary finite ordered set. For the ordered set $P$ in Figure 7 both $L_{1}$ and $L_{2}$ are optimal linear extensions: $s\left(P, L_{1}\right)=s\left(P, L_{2}\right)=s(P)=2$. Still neither $L_{1}$ nor $L_{2}$ can be constructed from the other by any sequence of linear extensions each constructed by interchanging chains. Nonetheless, at this writing there is no counterexample to this conjecture. For any finite ordered set $P$ and any $L \in \mathcal{L}(P)$ there is an integer $k$ and a sequence of linear extensions $L=L_{1}, L_{2}, \ldots, L_{k}$ such that each $L_{i}$ is obtained from $L_{i-1}$ by interchanging chains and $s(P, L)=s\left(P, L_{1}\right) \geqslant s\left(P, L_{2}\right) \geqslant$ $\cdots \geqslant s\left(P, L_{k}\right)=s(P)$.

\section{REFERENCES}

G. Chaty and M Chein (1979), Ordered matchings and matchings without alternating cycles in bipartite graphs, Utilitas Math. 16, 183-187.

M. Chein and M. Habib (1980), The jump number of dags and posets: an introduction, Ann. Discrete Math. 9, 189-194.

O. Cogis (1982), Problem 4.6, Ordered Sets (I. Rival, ed.), Reidel, Dordrecht, p. 814. 
O. Cogis and M. Habib (1979), Nombre de sauts et graphes série-parallèles, RAIRO Inform. Théor. 13, 3-18.

R. P. Dilworth (1950), A decomposition theorem for partially ordered sets, Ann. of Math. (2) 51, 161-166.

D. Duffus, I. Rival and P. Winkler (1982), Minimizing setups for cycle-free ordered sets, Proc. Amer. Math. Soc. 85, 509-513.

G. Gierz and W. Poguntke (1982), Minimizing setups for ordered sets: a linear algebraic approach, SIAM J. Algebraic Discrete Methods.

P. Grillet (1969), Maximal chains and antichains, Fund. Math. 65, 157-167.

B. Leclerc and B. Monjardet (1973), Orders “C. A.C.”, Fund. Math. 79, 11-22.

W. R. Pulleyblank (1982), On minimizing setups in precedence constrained scheduling, Discrete Applied Math.

E. Szpilrajn (1930), Sur l'extension de l'ordre partiel, Fund. Math. 16, 386-389.

J. Valdes (1978), Parsing flowcharts and series-parallel graphs, Technical Report STAN-CS-78-682, Stanford.

J. Valdes, R. E. Tarjan and E. L. Lawler (1982), The recognition of series-parallel digraphs, SIAM J. Comput. 11, 298-313.

Department of Mathematics, The University of Calgary, Calgary, Alberta, Canada T2N 1N4 